\title{
Perfil competitivo como herramienta para la gestión estratégica de la investigación en universidades
}

\author{
WENDOLIN SUÁREZ-AMAYA* \\ JAZMÍN DÍAZ-BARRIOS** \\ MORELA PEREIRA BURGOS***
}

Artículo de reflexión sobre el desarrollo de ventajas competitivas por parte de las instituciones universitarias en el área de la investigación.

Recibido: 1 de mayo de 2020 · Evaluado: 28 de julio de 2020 - Aceptado: 31 de julio de 2020

Citar como: Suárez-Amaya, W., Díaz-Barrios, J. y Pereira, M. (2021). Perfil competitivo como herramienta para la gestión estratégica de la investigación en universidades. Hallazgos, 18(35), 259-286. DoI: https://doi.org/10.15332/2422409X.5768

* Doctora en Ciencias Sociales, con mención en Gerencia. Profesora-investigadora de la Facultad de Ciencias Empresariales de la Universidad Arturo Prat (Chile) y de la Facultad de Ciencias Económicas y Sociales (FCES) de la Universidad del Zulia (Venezuela).

Correo electrónico: wendolin.suarez@gmail.com ORCID: https://orcid.org/0000-0003-3825-5781

** Posdoctora en Gerencia. Profesora titular de la Facultad de Ciencias Económicas y Sociales (FCES) de la Universidad del Zulia (Venezuela) y de la Global Humanistic University (GHU) (Curaçao).

Correo electrónico: jazdibar.consultora@gmail.com ORCID: https://orcid.org/0000-0001-7569-8106

*** Doctora en Ciencias Sociales, con mención en Gerencia. Profesora titular de la Facultad de Ciencias Económicas y Sociales (FCES) de la Universidad del Zulia (Venezuela).

Correo electrónico: morela.pereira@gmail.com

ORCID: https://orcid.org/0000-0003-4331-1530 


\section{Resumen}

Dirigir estratégicamente es lograr una ventaja competitiva y mantenerla. Para ello, la matriz de perfil competitivo (MPC), basada en los factores claves de éxito (FCE), permite posicionarse frente a los competidores. En ese sentido, el objetivo de esta reflexión es plantear una herramienta gerencial para orientar el desarrollo de ventajas competitivas en investigación universitaria, teniendo en cuenta los FCE identificados en una investigación previa (investigadores, recursos, visibilidad y modelo de desarrollo). Se recaba información a través de revisión teórica de expertos en planificación estratégica, y se procesa a través de análisis de contenido y hermenéutica. El estudio revela que la aplicación de una MPC serviría para que cada institución desarrolle su perfil competitivo, y basado en este diseñe un plan estratégico — de manera que fortalezca los FCE, en los cuales tiene liderazgo, y desarrolle los que requieren mayores recursos- Incluir esta herramienta en la planificación estratégica de las instituciones académicas permitiría orientarlas permanentemente al éxito, ya que podría llevar a destinar los recursos hacia los factores clave que garantizarán sus objetivos de liderazgo regional en investigación y visibilidad mundial. Además de esto, podrían adoptar las mejores prácticas de aquella universidad o universidades que las superan en un determinado factor.

Palabras clave: Factores claves de éxito; Gestión estratégica; Perfil competitivo; Universidad competitiva. 


\title{
Competitive profile as a tool for strategic management of research in universities
}

\begin{abstract}
To manage strategically is to achieve a competitive advantage and to maintain it. For this purpose, the competitive profile matrix (CPM), based on the key success factors (KSF), allows to position oneself against competitors. In this sense, the aim of this reflection is to propose a management tool to guide the development of competitive advantages in research at universities, taking into account the KSF identified in previous research (researchers, resources, visibility and development model). Information is collected through theoretical review by experts in strategic planning, and processed through content analysis and hermeneutics. The study reveals that the application of a CPM would serve for each institution to develop its competitive profile, and based on this, design a strategic plan -in order that it strengthens the KSFS in which it has leadership and develops those that require greater resources. Including this tool in the strategic planning of academic institutions would make it possible to guide them permanently towards success, since it could lead to allocating resources to the key factors that will ensure their objectives of regional leadership in research and global visibility. In addition, they could adopt the best practices of that university or universities that surpass them in a certain factor.
\end{abstract}

Keywords: Key success factors; Strategic management; Competitive profile; Competitive university.

\section{Perfil competitivo como ferramenta para a gestão estratégica da pesquisa em universidades}

\section{Resumo}

Dirigir estrategicamente é obter uma vantagem competitiva e mantê-la. Para isso, a matriz de perfil competitivo (MPC), baseada nos fatores-chave do sucesso (FCS), permite o posicionamento diante dos concorrentes. Nesse sentido, o objetivo desta reflexão é apresentar uma ferramenta gerencial para orientar o desenvolvimento de vantagens competitivas em pesquisa em universidades, considerando os FCS identificados em uma pesquisa prévia (pesquisadores, recursos, visibilidade e modelo de desenvolvimento). A informação foi coletada por meio de análise de conteúdo e hermenêutica. $O$ estudo revela que a aplicação de uma MPC serviria para que cada instituição desenvolvesse seu perfil competitivo e, baseado nele, elaborasse um plano estratégico - de maneira que fortalecesse os FCs, nos quais tem liderança, e desenvolvesse os que requerem maiores recursos. Incluir essa ferramenta no planejamento estratégico das instituições acadêmicas permitiria orientá-las de forma permanente ao sucesso, já que poderia levar a destinar os recursos aos fatores-chave que garantissem seus objetivos de liderança regional em pesquisa e visibilidade mundial. Além disso, poderiam adotar as melhores práticas $\mathrm{da}(\mathrm{s})$ universidade(s) que a superam em um determinado fator.

Palavras-chave: Fatores-chave de sucesso; Gestão estratégica; Perfil competitivo; Universidade competitiva. 


\section{Introducción}

Cada vez es más común la adopción y el uso de términos de índole empresarial en la academia. La universidad competitiva y el posicionamiento que le otorgan a su país de origen forman ahora parte del lenguaje cotidiano. Todo ello viene aparejado a los procesos de globalización y al modelo de desarrollo basado en el conocimiento, y ambos elementos tienen repercusiones en las políticas y acciones de la educación superior, con su consecuente introducción en la competitividad global (López-Leyva, 2016).

Este cambio se expresa en el auge que en las últimas décadas ha tomado la cultura de evaluación en las instituciones de educación superior, y en la reputabilidad como parte de la cultura de calidad y éxito de las instituciones (Mora et ál., 2015), por citar solo algunos de los fenómenos asociados. En este escenario, la investigación aparece como un elemento estratégico ligado al concepto de universidad exitosa. Tal como señala Connell (2019), el prestigio de las universidades en el mundo depende en gran medida del trabajo de investigadores; sus descubrimientos iluminan hacia nuevas tecnologías, profundizan nuestra comprensión del mundo y guían el trabajo de profesiones.

Señala Páez (2010) que "el fortalecimiento de las instituciones de educación superior en América Latina pasa por entender uno de sus procesos más importantes y del cual se sostienen todas las demás actividades universitarias: la investigación” (p. 1). Por esto, las universidades requieren hacer uso de herramientas de gestión que les permitan identificar su situación en el contexto que las rodea. La Matriz de Perfil Competitivo (MPC) aporta esa posibilidad, y más allá de eso, permite revisar las estrategias utilizadas y determinar hasta qué punto están apuntando hacia el éxito o no.

El estado del arte remite a variedad de trabajos que identifican factores claves de éxito (FCE) para las universidades en diversas aristas, entre las que se pueden citar la relación universidad-sector externo (Schofield, 2013; Plewa et ál., 2013; Herbert et ál., 2018) y los FCE basados en la idea de universidad emprendedora (Rice et ál., 2010; Choi y Park, 2013). Por su parte, Peláez (2016) identificó un listado de FCE y la consecuente matriz para universidades en Filipinas. Estos trabajos sirven de referente para desarrollar análisis de MPC en universidades.

En atención a lo anterior, esta investigación documental se centró en ofrecer a la comunidad científica y académica una herramienta gerencial para orientar el desarrollo de ventajas competitivas en investigación, basadas en el modelo de planeación estratégica, a través de los factores claves de éxito y la matriz de perfil competitivo. 
Para esto se trabajó desde los FCE planteados por Suárez y Díaz-Barrios (2014), desarrollando los indicadores de cada factor y construyendo una matriz que permita comparar cada universidad con sus pares nacionales. La investigación fue documental, utilizando el método hermenéutico y el análisis de contenido como técnica para el abordaje de los documentos.

\section{Planeación estratégica y perfil competitivo}

La tendencia de la sociedad globalizada a una creciente complejidad, aunada al tránsito de la modernidad a la posmodernidad, muestran al ser humano en un contexto en el que los cambios parecen haber tomado un ritmo cada vez más acelerado, y ponen en evidencia la madeja de redes que conectan a los grupos sociales en una relación de interacciones recíprocas, que, si bien mantienen un orden económico, político, social y cultural, entre otros, a la par conducen a procesos de inestabilidad y caos. La globalización es, entonces, un fenómeno no solo económico sino social, que muestra la propensión hacia una mayor integración e interdependencia entre los diversos países, lo que implica nuevos retos y complejidades (Camisón y Dalmau, 2009).

Este es el nuevo orden global en el cual operan las organizaciones sometidas a un entorno de permanente cambio, con la consecuente inestabilidad y requerimiento de ajuste continuo, lo cual dificulta la posibilidad de prever el comportamiento de las variables que las rodean. El cambio como constante exige un proceso de toma de decisiones apalancado en un pensamiento estratégico, el cual implica para Garrido (2014) "la capacidad de pensar en torno a un objetivo central con orientaciones de largo plazo” (p. 4). Además, tal como plantea Mintzberg (2009), el pensamiento estratégico no se limita a un repertorio en el que cabe todo lo referido a la gestión estratégica; es una manera singular de ver las cosas, con elementos que la hacen diferenciable de otras formas de gestión.

Pensar estratégicamente conlleva una visión sistémica que se decanta por la totalidad frente a la particularidad. En términos de Garrido (2014), se potencia una función definida como big picture analysis, que parte de la desagregación de los componentes del fenómeno para su comprensión inicial hacia una visualización global o de conjunto. Esto supone, además, pasar de lo obvio a lo que no se aprecia a simple vista, sino que se percibe mejor desde una mirada intuitiva.

Una herramienta que permite poner en acción el pensamiento estratégico es la planificación estratégica, entendida por Garrido (2014), como: 
Patrón sistemático y metódico que permite el desarrollo de una visión de largo plazo [...] y de objetivos ambiciosos [...]; identificar incertidumbres centrales [...]; detectar suposiciones críticas, verificarlas, contrastarlas [...]; convertir datos en información confiable; evaluar en forma realista la empresa de hoy y sus potencialidades; equilibrar la relación entre crecimiento, sumada a la investigación y desarrollo e innovación (I+D+i), con la operación actual; planificar coherentemente con la visión y la misión definida por la empresa; incorporar a todos los implicados en ejecutar e involucrar a todos los afectados en la elaboración. (p. 6)

Interpretando a Garrido (2014), se puede afirmar que la posición que la organización alcance a largo plazo está determinada no solo por la puesta en marcha de la planificación estratégica, sino por la incorporación del pensamiento estratégico como base de la gestión. En otras palabras, implica asumir cambios que afectan la esencia misma de la organización, y sus repercusiones tendrán efecto tanto en las prácticas directivas y operativas como en el clima y la cultura de la entidad.

Para David (2008), la planificación estratégica se concibe como la toma de decisiones o selección de medios para la concreción de objetivos, en tres grandes momentos: formulación, implantación y evaluación, todo ello con la intermediación de las funciones organizacionales. Desde esta óptica, la organización como entidad es una totalidad, en la cual las diversas funciones que la componen participan de manera conjunta en la configuración de un plan de largo plazo, del cual son copartícipes en la ejecución y los resultados.

El enfoque estratégico de la planificación, configurado como modelo genérico, sigue, según Koontz et ál. (2012), las siguientes etapas: 1) análisis de la industria o evaluación de su atractivo en el mercado; 2) perfil empresarial, que implica la determinación de valores, orientación ejecutiva, visión, propósitos, objetivos, entre otros; 3) desarrollo de estrategias alternativas a partir del análisis de los factores internos y externos; 4) evaluación y decisión estratégica; 5) instrumentación de la estrategia; 6) dirección-control; y 7) prueba de congruencia entre el objetivo y la estrategia y planeación de contingencias.

Retomando a David (2008), la planificación estratégica comprende tres etapas principales: la primera de ellas es la formulación, que comprende la declaración de la misión y visión del negocio, las auditorías externas e internas, el establecimiento de objetivos a largo plazo y la creación, evaluación y selección de la estrategia; luego de ello, las otras dos etapas corresponden a la implementación y la evaluación de la estrategia. 
Un aspecto fundamental de la planificación estratégica es sin duda la consideración de la competencia como factor determinante en la configuración de las estrategias organizacionales. En ese sentido, cobra fuerza el concepto de factores claves de éxito como aquellas funciones, actividades o prácticas que hacen a una empresa en determinada industria ser exitosa en el mercado con respecto a sus competidoras. Los FCE son, generalmente, comunes para todas las organizaciones de una industria específica, pero el desempeño en cada factor es lo que distingue a una empresa de sus competidoras, y conocer esos factores permite orientar el desarrollo de una competencia distintiva que la separe del promedio.

En este sentido, para el clásico Ohmae (1982), los FCE son potencialmente generadores de ventajas competitivas al viabilizar el desarrollo de habilidades y conocimientos que pueden distinguir a la organización, lo que los convierte en una fortaleza. Son factores vistos también por el cliente como valiosos o que proporcionan a las empresas una ventaja significativa en términos del costo. Estos factores varían de una industria a otra y, como se dijo líneas atrás, generalmente en una misma industria son los mismos, aunque se dan casos especiales en los cuales en una misma industria varían para una organización (Johnson et ál., 2011).

Diferentes autores tienen distintas lecturas para los FCE. Por ejemplo, Blanco (2000), Beltrán (1998) y Zambrano (2006) los visualizan como elementos internos de la organización que definen los resultados de la gestión, de ahí deriva la necesidad de controlarlos. Estos autores asumen la corriente de recursos y capacidades planteada en principio por Rockart (1979). Blanco llega incluso a entenderlos como el tercer elemento en el proceso, después de la definición de los objetivos y la filosofía de la organización, ya que en ellos debe centrarse el control de la gestión, mientras que Beltrán (1998) habla de ellos como los indicadores necesarios para mantener ese control.

Otros autores como David (2013) o Thompson et ál. (2012) van más allá y los presentan además como los elementos determinantes en los cuales se debe superar a la competencia para tener éxito en un mercado. En un proceso de planificación estratégica, plantea David (2013), los análisis externo e interno de una organización, así como la definición de fortalezas y debilidades, pasan por precisar los FCE de la industria analizada. Es decir que a partir del análisis externo (fuerzas económicas; fuerzas sociales, culturales, demográficas y ambientales; fuerzas políticas, gubernamentales y legales; fuerzas tecnológicas; fuerzas competitivas) y el análisis interno (áreas funcionales, dependiendo del tipo de industria), se podrán hallar los FCE para, a partir de ahí, trabajar en competencias distintivas que deriven en ventajas competitivas y en la consiguiente sostenibilidad de la organización. 
Resumiendo, los FCE son un denominador común que las distintas organizaciones de una industria deben perseguir y orientar hacia el más elevado desempeño, porque comportan los elementos que el mercado precia para favorecer a una determinada organización y convertirla en un éxito sustentable. Ahora bien, para identificar la calificación que la organización estudiada y sus competidoras tienen en estos factores, la planificación estratégica se sirve de análisis competitivos que permiten comparar el desempeño de acuerdo a elementos objetivos; David (2013) agrupa estos análisis en una matriz de perfil competitivo, mientras que Thompson et ál. (2012) los entienden como una evaluación de la fortaleza competitiva general.

La MPC es una herramienta que ayuda a las empresas a evaluarse a sí mismas frente a sus competidores importantes, para lo cual incorpora los FCE de esa industria (Zimmerer et ál., 2008). Según D’Alesio (2008), la MPC se basa en los FCE que resultan comunes al grupo de competidores y, adicionalmente, son determinantes o críticos para alcanzar el éxito en el ámbito de actuación de las organizaciones involucradas. Plantea este autor que la construcción de la MPC involucra la participación de todos los posibles competidores.

En términos de David (2011), la MPC permite identificar los rivales destacados de una empresa, incluidos sus puntos fuertes y débiles, en comparación con la posición estratégica de otra empresa tomada como referencia. Con esta información es posible decidir sobre estrategias sustentadas en el posicionamiento de los rivales en el sector o definir cuáles estrategias emplear. Así como la determinación de los FCE es un proceso netamente cualitativo, de análisis, el posicionamiento con respecto a otros es más bien cuantitativo, a partir de criterios y percepciones cualitativas basadas en la experiencia y el conocimiento de quienes lo desarrollan.

Para elaborar la MPC, siguiendo a David (2008), se debe: 1) hacer una lista de FCE, sin discriminar si el factor es externo o interno, debido a que se trata de un listado de factores clave no inherentes a la organización sino al sector; 2) estipular para cada factor un peso relativo, desde 0,0 (no importante) a 1,0 (muy importante), que indique la preponderancia del factor para alcanzar el éxito en el sector donde se participa; la suma de todos los pesos debe ser la unidad; 3) calificar cada FCE entre 1 y 4 puntos para medir hasta qué punto las estrategias responden al factor de manera eficaz, pues a mayor puntuación mayor eficacia, y viceversa; 4) multiplicar el peso asignado a cada factor por la puntuación estipulada, a fin de tener un resultado ponderado; 5) sumar las calificaciones obtenidas para determinar el total ponderado de la organización. El resultado ponderado más alto que se puede alcanzares 4,0, siendo 
el más bajo 1,0, mientras que el promedio es 2,5. Otros autores como Thompson et ál. (2012) sugieren escalas diferentes, incluso no numéricas.

En la misma línea de pensamiento, Zimmerer et ál. (2008) establecen tres pasos en la construcción de la MPC para una empresa. El primer paso es encontrar los FCE y asignar el peso a dichos factores de acuerdo con su importancia relativa. En el siguiente paso, la empresa debe identificar a sus principales competidores y calificarlos, en cada uno de los FCE (incluyendo la organización que es objeto original del análisis). Por último, se multiplica el peso por la calificación para cada factor, para obtener una puntuación ponderada, y luego se suma la puntuación ponderada de cada competidor y así se obtiene una puntuación ponderada total.

La auditoría externa realizada a través de la MPC ha pasado a ser un subproceso esencial dentro del proceso global de la planificación estratégica, por lo que resulta imperativo facultar al personal, tanto gerencial como operativo, en la tarea de evaluar el entorno y sus fuerzas externas clave, de modo tal que sean capaces de anticipar las oportunidades y amenazas nacientes y, en consecuencia, seguir estrategias eficaces que potencien el desarrollo de la organización (David, 2008).

Si bien los lineamientos teóricos planteados en cuanto a la planificación estratégica parecen ser aplicables particularmente al ámbito empresarial, lo cierto es que tienen aplicación general, tanto en el ámbito privado como público, de producción o de servicios. En ese sentido, el análisis de los FCE y la elaboración de una MPC particular derivan de la especificidad del sector de que se trate. Uno de estos sectores es el académico, específicamente las universidades, que, como toda organización, buscan destacarse y ser valoradas en este mundo global, principalmente a través de una de sus funciones básicas como es la investigación, la cual tiene la cualidad de la visibilidad que potencialmente puede catapultar organizaciones dedicadas al conocimiento hacia el renombre mundial.

\section{Factores claves de éxito de la investigación universitaria}

En esta aldea comunicacionalmente global donde los rankings universitarios se evalúan anualmente y ubican a cada universidad en una posición de acuerdo con su desempeño, conocer las maneras de elevar el desempeño en investigación se convierte en un imperativo. En este sentido, Suárez y Díaz-Barrios (2014) identificaron los FCE para la investigación universitaria, en el entendido de que son los elementos primordiales “para alcanzar el éxito organizacional que son controlables por la gestión” (p. 112). 
En su investigación, las mencionadas autoras consultaron a investigadores de larga trayectoria y a actores gerenciales de los distintos entes involucrados en el proceso investigativo, en cada una de las cuatro universidades venezolanas con mejor desempeño y mejor ubicadas en los rankings latinoamericanos. De esta manera identificaron cuatro factores que consideran que influyen directamente en el logro de objetivos y el consiguiente éxito de una universidad: "investigador, recursos, visibilidad y modelo de desarrollo" (p. 115) de la universidad de que se trate. Las autoras enuncian a su vez veinticuatro indicadores de desempeño que representan un primer camino a seguir para, inicialmente, ubicarse en el espectro nacional a través de la MPC y, en segundo lugar, desarrollar características distintivas, y con ellas, ventajas competitivas con respecto a sus pares.

\section{El investigador}

Para Suárez y Díaz-Barrios (2014), el investigador en sí mismo, como protagonista del proceso, es el primer FCE de la investigación. Las autoras plantean cinco aspectos a los cuales debe prestarse atención: número de investigadores e incremento anual; número de doctores e incremento anual; programas de estímulo individual y sus resultados; programas de estímulos colectivos y sus resultados; creación de patentes.

El primer aspecto implica en principio la creación y el seguimiento de indicadores que monitoreen tanto el número de investigadores activos como el incremento anual de ellos; aunque también implica recíprocamente el desarrollo de una política de apoyo a la investigación dentro de la carrera académica, para incentivar tanto el incremento de investigadores como el crecimiento de los ya existentes. Debe ser tan atractivo para un académico novel incursionar en la investigación, como para un investigador consolidado permanecer en ella; no solo en el aspecto económico, sino también en prestigio, posibilidades de formación y orientación de carrera; con una evaluación profesional basada, no únicamente en la docencia, sino también en su producción intelectual.

En el enfoque de las políticas orientadas al incremento de investigadores se tiene el segundo aspecto: la formación de doctores, dado que los programas de doctorado (Ph. D.) representan el espacio fundamental de donde surgen la mayoría de los investigadores. Explican las autoras que el incremento de investigadores pasa por el desarrollo de planes para la formación de quinto nivel. Esta percepción es apoyada por Canales (2011), quien expresa que uno de los principales elementos para asentarse como universidad de investigación de clase mundial es elevar el nivel de escolaridad del personal y mejorar su tiempo de dedicación. Estos planes pueden ser 
desarrollados desde los centros de investigación o desde las facultades de adscripción, con apoyo financiero de la universidad, los entes gubernamentales nacionales $\mathrm{u}$ organismos multilaterales que ofrezcan programas con oportunidad desde becas.

Otro indicador del factor clave investigadores lo constituyen los programas de estímulo a la investigación, tanto individuales como colectivos. Canales (2011) señala que en México el programa de estímulo ha logrado incrementar significativamente tanto el número de investigadores como la investigación en sí misma. Añade, que ha contribuido además a la des-homologación del trabajo académico a través de las asignaciones salariales a los investigadores productivos.

Los programas individuales influyen en la motivación del investigador al premiar su esfuerzo y productividad científica, reconociendo el impacto que los resultados de las investigaciones realizadas tienen tanto en la universidad como en la comunidad científica nacional e internacional y la sociedad. Estos programas de estímulo pueden concretarse tanto con suplementos salariales como con posibilidades de becas para formación de cuarto y quinto nivel, asistencia financiada a eventos de difusión científica, publicación de libros sin costo para el académico, entre otros.

Los programas de estímulo colectivo (para los centros, los institutos, las facultades o las universidades más destacadas) crean y cohesionan equipos de trabajo, promueven políticas internas con beneficios para los equipos de investigación y los investigadores, y desarrollan una ventaja competitiva para la unidad de investigación, facultad o universidad beneficiada, diferenciándola de sus pares.

Ambas modalidades contribuyen a incrementar el número de investigadores en las distintas universidades; prueba de ello son los resultados de los programas promovidos desde 1984 en México (Sistema Nacional de Investigadores [SNI]) y en Venezuela entre 1990 y 2010 (Programa de Promoción al Investigador [PPI]) (Marcano y Phélan, 2009). En ambos casos se elevó sustancialmente el número de académicos dedicados a la investigación, lo que significó una ventaja competitiva para estos países durante el periodo de vigencia.

El último elemento a considerar es la creación de patentes. Estas protegen las invenciones, son una medida de innovación y competitividad de los países y regiones, y representan un elemento importante en los indicadores de las universidades. Sin embargo, hasta el momento los países latinoamericanos tienen un pobre desempeño en esta área. Luzardo (2019) afirma que la falta de inversión por parte de los Estados, el desconocimiento legal y la desconfianza en los sistemas de propiedad intelectual que existen en América Latina y el Caribe son factores que se conjugan para que las 
cifras de patentes sean absurdamente bajas en la región. Ni en las universidades ni en los países hay apoyo para el registro de patentes, de modo que las pocas iniciativas que se generan tienden a registrarse en el exterior, donde sí existe confianza en lo que respecta a legalidad, reconocimiento y visibilidad.

\section{Recursos financieros}

El segundo factor definido en el trabajo de Suárez y Díaz-Barrios (2014) fue recursos, aun cuando podría hablarse más específicamente de recursos financieros. Resulta claro que para desarrollar y mantener la investigación con un alto desempeño se requiere contar con una cantidad de recursos necesarios y suficientes. En ese sentido, en el documento referido, se acotan seis elementos: asignación específica para investigación desde los gobiernos nacionales; generación de ingresos propios; dotación de infraestructura, mobiliario, equipo y materiales; acceso a sistemas de información internacional; bibliografía actualizada; y soporte legal para la propiedad intelectual.

Con respecto al financiamiento gubernamental, Xue et ál. (2004) explican que China, un país con un claro ascenso en investigación en el presente siglo, ha estimulado a los institutos de educación superior para que trabajen plenamente en la investigación científica con gran cantidad de fondos, a fin de que se conviertan en la base de la investigación científica fundamental de esa nación. Por otra parte, en Alemania existen dos programas: Clúster de excelencia y Universidades de excelencia, financiados en un $75 \%$ por el gobierno nacional y un $25 \%$ por el regional (del lugar donde se encuentre la universidad), respectivamente, para fortalecer y fomentar la investigación de alto nivel en las universidades y retener a los mejores investigadores en estas (Comisión Europea, 2019).

Los dos casos anteriores son ejemplo de la importancia que países del primer mundo dan al financiamiento de la investigación como vía para desarrollar universidades de clase mundial, con innovación y tecnología. Pero no solo se trata de este tipo de financiamiento, ya que otro indicador requerido es la generación de ingresos propios, que garantizan a la investigación una mayor independencia y permanencia en el tiempo, incluso con problemas de financiación gubernamental. Desarrollar este indicador requiere una adecuada relación entre universidad y empresa con fines de beneficio mutuo (ganar-ganar): las empresas salen beneficiadas a través del desarrollo de tecnología para sus procesos, y la universidad por las posibilidades de autofinanciamiento que esta colaboración representa. Adicionalmente, algunos rankings, como Times Higher Education (THE, 2020), consideran entre sus indicadores los ingresos que la universidad recibe de la industria, vale decir, el pago por 
servicios que las empresas hacen a las universidades por la transferencia de conocimientos desde la investigación.

La existencia de recursos implica financiamiento de proyectos de investigación, dotación de laboratorios, contratación de ayuda técnica, adquisición de equipos, registro y mantenimiento, así como suscripciones internacionales a bases de datos y revistas, financiamiento de congresos, publicaciones y cursos de actualización, e incluso incentivos a los investigadores, equipos de investigación y centros o institutos de investigación. Por el contrario, no contar con los recursos necesarios hace incierto el destino de la investigación, en la medida en que, según Colina (citado por Suárez y Díaz-Barrios, 2014), esta situación amenaza "la continuidad de proyectos de investigación, la oportuna publicación de las revistas arbitradas, la asistencia a eventos científicos nacionales e internacionales e incluso la eliminación de premios (incentivos monetarios) que algunas universidades manejan para investigadores productivos” (p. 114).

\section{Visibilidad}

El tercer FCE definido fue la visibilidad, entendida en palabras de Corchuelo (2018) como la "capacidad de hacer disponible la producción intelectual a la comunidad científica a través de diversos medios y soportes” (p. 78). Para este autor, la visibilidad no necesariamente se traduce en incremento de citas que impactan los rankings, pero sí en "un aumento directo en la utilización de los contenidos producidos” (p. 78). En este caso, los indicadores de desempeño son siete: apoyo a la publicación; creación de repositorios institucionales; ponencias en eventos científicos nacionales e internacionales; organización de eventos científicos; desarrollo y mantenimiento de rankings nacionales; posicionamiento en rankings universitarios; creación y participación en redes de conocimientos.

El apoyo a la publicación, bien sea editando revistas o apoyando al investigador para que publique en medios internacionales de alto impacto y en otros idiomas, eleva la posibilidad de que los resultados de los investigadores sean leídos fuera de sus universidades y países. La organización de eventos y presentaciones en eventos nacionales e internacionales garantiza la necesaria retroalimentación con pares de la comunidad científica y grupos de interés. La creación de repositorios, rankings y redes facilita el intercambio de la producción científica y visibiliza más a las instituciones. Esto último está alineado con los criterios que los rankings internacionales toman en cuenta para evaluar y, por ende, posicionar a las universidades. Dado que las publicaciones y citas recibidas son un factor tan relevante, incrementar la 
posibilidad de publicación de los investigadores es determinante para el posicionamiento de la universidad de adscripción.

Como telón de fondo de cada FCE analizado, y de este en particular, aflora la necesidad de políticas institucionales que hagan posible el desarrollo positivo de indicadores. Visibilidad implica un esfuerzo de la universidad para que la producción intelectual de cada investigador esté disponible y pueda ser consultada por sus pares, y también que ese investigador pueda nutrirse de otros conocimientos, ya sea asistiendo a eventos u organizándolos en su propia universidad. Todo esto comporta un importante esfuerzo apalancado por políticas públicas. En otras palabras, las universidades que lideran mundialmente la creación de conocimiento lideran igualmente publicaciones, bases de datos, eventos, redes; en Latinoamérica, estas se han desarrollado a través del apoyo de serias políticas de Estado, como lo hizo, y continúa haciendo, Brasil.

\section{Modelo de desarrollo}

El cuarto factor encontrado tiene que ver con la formulación de un modelo de desarrollo estratégico de la investigación que oriente de la mejor forma la investigación en cada institución en particular. Dicho modelo debe identificar las áreas en las cuales la investigación está más adelantada, para impulsarla, sin que esto signifique descuidar las otras. La idea es evaluar las capacidades reales de desarrollo de un área en particular (salud, ciencias sociales, humanidades, ciencias básicas, tecnología, entre otros) y orientar los esfuerzos para catapultarla a escala nacional o internacional.

Este factor se asocia con seis indicadores de desempeño: un análisis FODA de la gestión de investigación en cada universidad, que permita conocer fortalezas y debilidades institucionales en materia de investigación; un plan estratégico de investigación a cinco años, construido a partir del análisis FODA, que ofrezca un objetivo y oriente el camino a seguir; un plan operativo anual, derivado del plan estratégico para cada unidad de investigación, que defina metas anuales y concretas a cumplir para la unidad como un todo y por los distintos equipos de investigación en ella insertos.

A lo anterior se añaden líneas de investigación claras, definidas, en las que los investigadores noveles puedan incluirse y los maestros consolidarse, que representen las capacidades distintivas y orienten los esfuerzos de la universidad. De igual manera se requiere definir áreas emergentes del conocimiento para que la investigación y la universidad se mantengan a la vanguardia en las fortalezas que poseen, es decir, mantengan sus ventajas competitivas. Por último, y reforzando otros FCE, 
se tienen los incentivos institucionales por desempeño de unidad de investigación basados en elementos de visibilidad.

En general, el modelo tiene que ver con "la necesidad de tomar en consideración las áreas del conocimiento que están más desarrolladas y/o aquellas que requieren concentrar esfuerzos” (Suárez y Díaz-Barrios, 2014, p. 115). Por citar un ejemplo, según datos gubernamentales, la mayor parte de la investigación que se desarrollaba en Venezuela en la primera década de este siglo estaba dirigida a las ciencias sociales $(35,8 \%)$, las ciencias agro-ambientales ( $22 \%)$ y biológicas-salud (20\%); otras áreas tenían porcentajes sensiblemente menores: ingenierías y tecnologías (12,9\%), ciencias básicas (9,7 \%) (Meléndez, 2010). Sin embargo, la investigación venezolana con impacto internacional es mayoritariamente en el área médica y de la neurociencia e inmunología, con un 30 \% (Scimago Country Rank, 2020).

Esto último podría significar un apoyo implícito a determinadas organizaciones con la suficiente capacidad, entorno, equipos y personal de alto nivel como para lograr presencia internacional. Tal vez, cada universidad debería especializar su investigación y orientar sus esfuerzos a las áreas particulares de mayor fortaleza que permitan desarrollar ventajas competitivas, presencia internacional y logro sostenido de los objetivos organizacionales.

Lo anterior no niega que de vez en cuando puedan aparecer grupos aislados de investigación con un desempeño brillante y alta productividad que logren el reconocimiento nacional e internacional, pero son anomalías del sistema, producto más bien del trabajo de sus miembros y no de un sistema que les dé soporte. No obstante, la existencia de un modelo definido de investigación podría convertir dicha anomalía en la pauta y hacer la diferencia para la universidad y para el país. En este sentido, los veinticuatro indicadores de desempeño planteados originalmente por Suárez y Díaz-Barrios (2014) fueron reinterpretados y ajustados a veintidós, los cuales, junto a los FCE, se presentan en la tabla 1.

Tabla 1. Factores claves de éxito e indicadores de desempeño de la investigación universitaria.

\begin{tabular}{|l|l|}
\hline Factores claves de éxito & \multicolumn{1}{c|}{ Indicadores de desempeño } \\
\hline \multirow{3}{*}{ Investigadores } & Número de investigadores activos e incremento anual \\
\cline { 2 - 2 } & Número de Ph. D. e incremento anual \\
\cline { 2 - 2 } & Programas de estímulo (individual y colectivo) \\
\cline { 2 - 2 } & Patentes registradas \\
\hline
\end{tabular}




\begin{tabular}{|c|c|}
\hline Factores claves de éxito & Indicadores de desempeño \\
\hline \multirow{5}{*}{ Recursos financieros } & Financiamiento gubernamental a la investigación \\
\hline & Generación de ingresos propios \\
\hline & Dotación de infraestructura, mobiliario, equipos y materiales \\
\hline & $\begin{array}{l}\text { Acceso a sistemas de información internacional y bibliografía } \\
\text { actualizada }\end{array}$ \\
\hline & Soporte legal para la venta de productos del conocimiento \\
\hline \multirow{7}{*}{ Visibilidad } & Apoyo a la publicación en revistas de alto impacto y otros idiomas \\
\hline & Repositorios institucionales y otras formas de acceso abierto \\
\hline & $\begin{array}{l}\text { Presentación de resultados en eventos científicos nacionales e } \\
\text { internacionales }\end{array}$ \\
\hline & Organización de eventos científicos nacionales e internacionales \\
\hline & Creación y mantenimiento de rankings nacionales \\
\hline & $\begin{array}{l}\text { Posición en clasificaciones de universidades latinoamericanas y } \\
\text { mundiales }\end{array}$ \\
\hline & Creación y mantenimiento de redes de conocimientos \\
\hline \multirow{6}{*}{ Modelo de desarrollo } & Matriz FODA de la gestión de investigación en cada universidad \\
\hline & Plan estratégico de investigación a cinco años en cada universidad \\
\hline & Plan operativo anual de cada unidad de investigación \\
\hline & $\begin{array}{l}\text { Orientación a la investigación en las líneas consolidadas } \\
\text { (capacidades distintivas) }\end{array}$ \\
\hline & Monitoreo de áreas emergentes del conocimiento \\
\hline & $\begin{array}{l}\text { Incentivos institucionales por desempeño basado en elementos de } \\
\text { visibilidad }\end{array}$ \\
\hline
\end{tabular}

Fuente: elaboración propia a partir de Suárez y Díaz-Barrios (2014). 


\section{Matriz del perfil competitivo en investigación para universidades}

La búsqueda de la excelencia es un ideal perseguido tradicionalmente por las organizaciones que buscan el conocimiento. Para las universidades, esto implicó en principio una búsqueda interna, en competencia consigo mismas. Sin embargo, la globalización de las comunicaciones cambió esto e hizo que las universidades comenzaran a competir dentro de los países, en Latinoamérica, Iberoamérica y el mundo por ese calificativo. Para Ballbé (1999), la competencia entre universidades es la esencia del sistema pluralista de enseñanza superior, además de proveer el mecanismo de afloramiento de nuevas ideas para desburocratizar las administraciones, generar dinamismo y realizar una evaluación comparativa entre los resultados de diferentes universidades.

Ello implica que las universidades deben contar ahora con una serie de datos que antes resultaban innecesarios, como por ejemplo: cuáles elementos las hacen más competitivas; qué hacen mejor o peor otras universidades que están en el mismo entorno y cuáles son esas universidades; cómo puede una universidad alcanzar a otra que tiene un mejor desempeño y mantener la distancia de otra que se encuentra por debajo. Estas son algunas preocupaciones de las universidades en cualquier país o regiones del mundo, pequeñas o grandes, especializadas o generales. Todas están en competencia, y todas quieren destacar.

Para ayudar en esta labor se acude a la planificación estratégica (elemento fundamental en una universidad que aspire a ser de clase mundial), y dentro de ella al análisis de fortaleza competitiva (Thompson et ál., 2012; David, 2008), herramienta empresarial que, como se mencionó, evalúa la fortaleza competitiva general de una organización, ubicándola en relación con sus competidoras e informando de sus propias ventajas o desventajas competitivas (Thompson et ál., 2012).

Entonces, en el ámbito particular de la investigación universitaria, ¿qué puede hacer esta herramienta para mejorar el desempeño de una institución particular? La respuesta es que el conocimiento propio y del oponente da, de entrada, una ventaja competitiva para actuar, para disminuir debilidades y mantener fortalezas. ¿Cómo se hace este proceso? Estos serían los pasos a seguir:

1. Precisar los FCE de la investigación universitaria. En tal sentido, en este artículo se presentan los cuatro factores y veintidós indicadores que apoyan a la universidad en dicho análisis. 
2. Analizar estos factores e indicadores y ubicar si son fortalezas o debilidades en la universidad. Listar todos los elementos y clasificarlos. Ejemplo: ¿es el número de doctores una fortaleza o una debilidad?, ¿es la dotación de infraestructura una fortaleza o una debilidad? Si se las ubica como fortalezas, entonces se debe definir si son ligera o verdaderamente fuertes en el área, y de igual manera se hace con las debilidades que se encuentren: ¿es una debilidad menor o una gran debilidad? Para la clasificación se pueden usar escalas de 1-4 o 1-8 (distintos autores utilizan distintas escalas), lo importante es que el análisis sea honesto y exhaustivo porque de este dependerá la calidad del análisis competitivo. Entiéndase que las escalas deben ser pares, porque para la universidad debe haber una clara definición respecto a si el aspecto analizado es una fortaleza o una debilidad, y las escalas impares permiten un punto ecléctico que no facilita la mejora del desempeño o la búsqueda de excelencia.

3. El tercer paso sería la identificación de las universidades competidoras. Primero se requiere decidir si se va a hacer la evaluación en un ámbito local, regional o nacional, para esclarecer cuáles serán las universidades seleccionadas. Para los criterios de selección de las universidades se propone tomar referentes específicos que permitan identificar a las instituciones competidoras en función de una serie de características que las hacen líderes en determinados campos de investigación. Una alternativa, tal como señalan Vera y Díaz (2012), sería seleccionar aquellas universidades con similitudes en producto ofrecido, mercado cubierto y capacidades estratégicas. Como guía se sugieren elementos tales como: cantidad de investigadores, número de publicaciones en revistas científicas, cantidad de proyectos de investigación, número de doctores, inclusión en determinado ranking, presupuesto devengado, entre otros.

4. Conocer a las instituciones competidoras en cada uno de los factores de éxito e indicadores, analizando la fortaleza o debilidad del indicador en particular y el FCE en general. En este sentido, David (2013) plantea que para formular una estrategia exitosa es fundamental recabar y evaluar exhaustivamente información sobre los competidores potenciales. Para ello, el autor señala el uso de inteligencia competitiva, definiéndola como "un proceso sistemático y ético para recabar y analizar información sobre las actividades y tendencias generales de la competencia con el propósito promover los objetivos propios de una compañía” (p. 74). Hay que servirse de múltiples fuentes, de manera que los datos sean vastos, actualizados y de calidad; esto, según el autor, "puede hacer diferencia 
entre tener un conocimiento superior sobre la competencia en general, o contar únicamente con información promedio” (p. 74). Lo importante aquí es mantener un flujo continuo de información estratégica y oportuna.

En el caso de la investigación universitaria, para establecer el puntaje por FCE de la competencia, en muchos países se cuenta con una gran cantidad de datos externos que proveen información bastante objetiva de la posición de cada universidad. Sin embargo, en general es recomendable utilizar los mejores y mayores datos de que se disponga (publicaciones, investigadores, rankings, premios, ingresos, redes), para hacerlo de la forma más objetiva posible. Aunque también, apelar al conocimiento del personal de investigación en las respectivas universidades competidoras y de informantes clave, con amplia visión de la investigación en el ámbito nacional e internacional, es acertado. Al fin y al cabo, la definición de estrategias tiene parte de arte y parte de ciencia. Vale decir que la calificación asignada a cada FCE puede variar en función tanto del contexto como de los recursos y las capacidades del sistema educativo en el cual se encuentre inmersa una universidad. Justamente por estas condiciones, la situación de cada una puede variar entre cada factor, y poner en condición ventajosa a una frente a otra.

5. El siguiente paso crucial es establecer la ponderación que en el contexto específico estudiado tienen los cuatro FCE. No en todos los contextos o grupos de universidades el investigador, la visibilidad, los recursos financieros o el modelo de desarrollo tendrán el mismo peso. Es necesario, entonces, analizar el contexto particular, sirviéndose de expertos, para definir los porcentajes de cada uno de los cuatro elementos que suman la totalidad. Esto se logra a través del conocimiento propio y de los competidores, volviendo la mirada al entorno para evaluar estos FCE y determinar la ponderación que les dan los grupos de interés de la investigación universitaria, al catalogar de exitosa a una universidad en ese entorno. Se trata sobre todo de determinar el peso específico que tienen el investigador, los recursos, la visibilidad y el modelo, en el entorno en el cual se trabaja. Una vez establecido el peso específico de cada factor, se hará lo propio con los indicadores del factor, vale decir, qué porcentaje representará cada indicador dentro del factor.

Con toda esta información, el siguiente paso es construir una matriz que permita visualizar de manera objetiva y numérica la posición que tanto la universidad como sus competidoras tienen en el entorno estudiado. Para ello, se 
listan en una columna los FCE, la ponderación de cada factor, la universidad estudiada y las universidades competidoras. Para construir la matriz, se multiplica la calificación asignada a cada institución por la ponderación dada a cada indicador.

A partir de los subtotales, se suman los resultados ponderados de cada indicador y se obtiene el resultado correspondiente al factor; sumando los valores de cada factor, se obtiene el puntaje de cada universidad. De esta manera se alcanza una visión general ponderada que permite establecer, numéricamente, las universidades con mejor desempeño general y con mejor desempeño en cada factor particular, así como la ubicación específica de la universidad estudiada. Se puede tener una idea de cuáles se desempeñan mejor que ella, sobre cuáles se tiene una ventaja competitiva y saber dónde están las fortalezas y las debilidades. Por su parte, los indicadores permiten conocer los elementos internos que definen un desempeño exitoso, y por ende, establecen la guía a seguir para lograrlo. En la tabla 2 se presenta la matriz construida a partir de los FCE e indicadores de desempeño identificados previamente.

Cabe señalar que el objetivo de esta matriz no es ofrecer resultados absolutos en cuanto a si una organización es mejor que otra, sino ofrecer información sobre cuál es el estado individual y comparativo de cada una con respecto a los factores considerados indispensables para lograr el éxito organizacional de la institución universitaria; al ser comparativa muestra quién tiene más o menos desarrollo en cada factor. Esta información permite a las instituciones conocer sus puntos fuertes y débiles, lo que deben mantener y lo que deben fortalecer o desarrollar, además de que da la oportunidad de emular las mejores prácticas de universidades que resultan superiores en un determinado factor. 
Tabla 2. Matriz del perfil competitivo para universidades líderes en investigación.

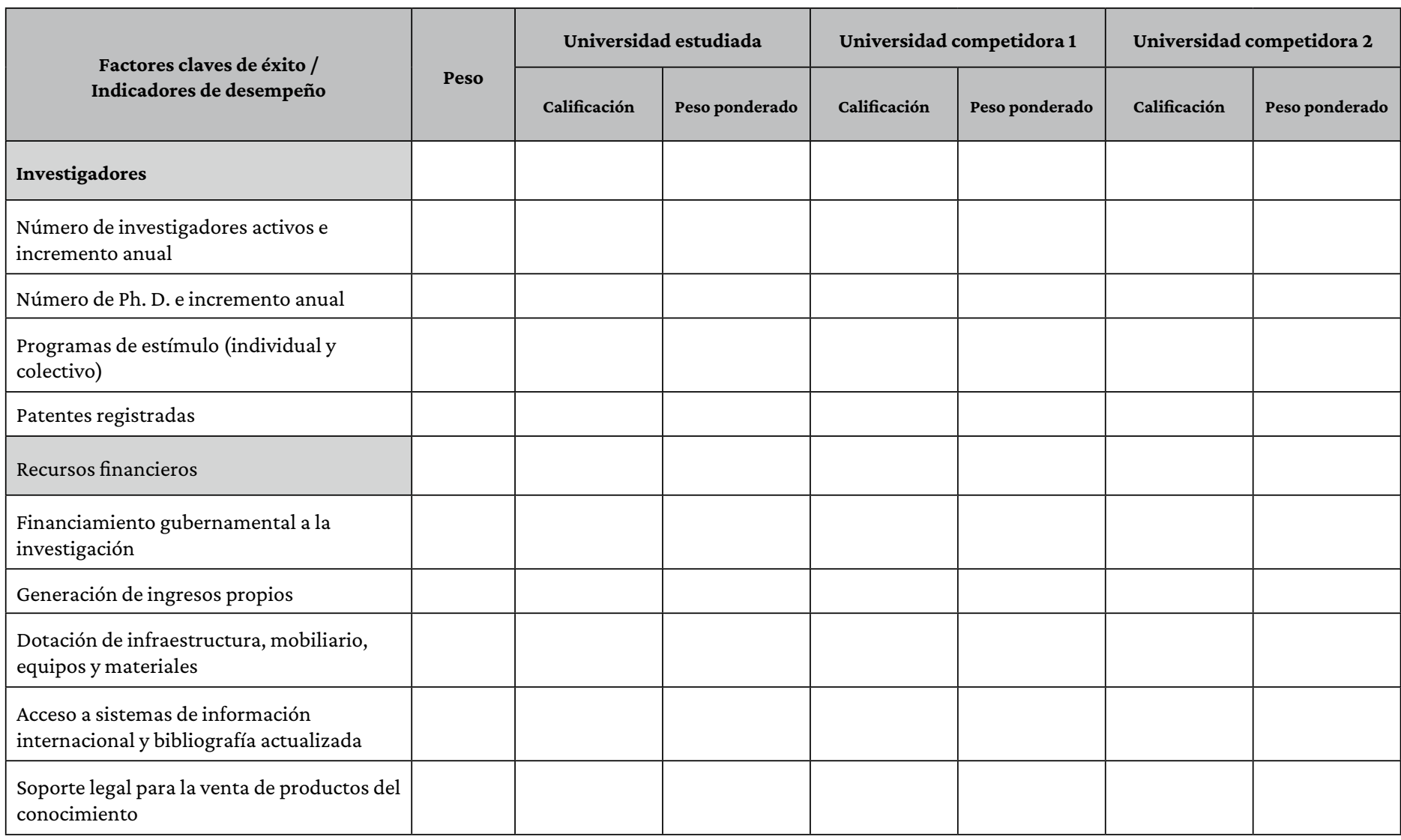




\begin{tabular}{|c|c|c|c|c|c|c|c|}
\hline \multirow{2}{*}{$\begin{array}{l}\text { Factores claves de éxito / } \\
\text { Indicadores de desempeño }\end{array}$} & \multirow{2}{*}{ Peso } & \multicolumn{2}{|c|}{ Universidad estudiada } & \multicolumn{2}{|c|}{ Universidad competidora 1} & \multicolumn{2}{|c|}{ Universidad competidora 2} \\
\hline & & Calificación & Peso ponderado & Calificación & Peso ponderado & Calificación & Peso ponderado \\
\hline Visibilidad & & & & & & & \\
\hline $\begin{array}{l}\text { Apoyo a la publicación en revistas de alto } \\
\text { impacto y otros idiomas }\end{array}$ & & & & & & & \\
\hline $\begin{array}{l}\text { Repositorios institucionales y otras formas } \\
\text { de acceso abierto }\end{array}$ & & & & & & & \\
\hline $\begin{array}{l}\text { Presentación de resultados en eventos } \\
\text { científicos nacionales e internacionales }\end{array}$ & & & & & & & \\
\hline $\begin{array}{l}\text { Organización de eventos científicos } \\
\text { nacionales e internacionales }\end{array}$ & & & & & & & \\
\hline $\begin{array}{l}\text { Creación y mantenimiento de rankings } \\
\text { nacionales }\end{array}$ & & & & & & & \\
\hline $\begin{array}{l}\text { Posición en clasificaciones de universidade } \\
\text { latinoamericanas y mundiales }\end{array}$ & & & & & & & \\
\hline $\begin{array}{l}\text { Creación y mantenimiento de redes de } \\
\text { conocimientos }\end{array}$ & & & & & & & \\
\hline Modelo de desarrollo & & & & & & & \\
\hline $\begin{array}{l}\text { Matriz FODA de la gestión de investigación } \\
\text { en cada universidad }\end{array}$ & & & & & & & \\
\hline
\end{tabular}




\begin{tabular}{|c|c|c|c|c|c|c|c|}
\hline \multirow{2}{*}{$\begin{array}{l}\text { Factores claves de éxito / } \\
\text { Indicadores de desempeño }\end{array}$} & \multirow{2}{*}{ Peso } & \multicolumn{2}{|c|}{ Universidad estudiada } & \multicolumn{2}{|c|}{ Universidad competidora 1} & \multicolumn{2}{|c|}{ Universidad competidora 2} \\
\hline & & Calificación & Peso ponderado & Calificación & Peso ponderado & Calificación & Peso ponderado \\
\hline $\begin{array}{l}\text { Plan estratégico de investigación a cinco } \\
\text { años en cada universidad }\end{array}$ & & & & & & & \\
\hline $\begin{array}{l}\text { Plan operativo anual de cada unidad de } \\
\text { investigación }\end{array}$ & & & & & & & \\
\hline $\begin{array}{l}\text { Orientación a la investigación en las líneas } \\
\text { consolidadas (capacidades distintivas) }\end{array}$ & & & & & & & \\
\hline $\begin{array}{l}\text { Monitoreo de áreas emergentes del } \\
\text { conocimiento }\end{array}$ & & & & & & & \\
\hline $\begin{array}{l}\text { Incentivos institucionales por desempeño } \\
\text { basado en elementos de visibilidad }\end{array}$ & & & & & & & \\
\hline Totales & & & & & & & \\
\hline
\end{tabular}

Fuente: elaboración propia. 


\section{Conclusiones}

La investigación universitaria está viviendo profundos cambios en la forma de medir sus resultados e impacto, por ello los indicadores y clasificadores se establecen actualmente como mecanismos para posicionar las instituciones. Si bien la definición de lineamientos para la acción mediante políticas públicas, el impulso ofrecido a partir de inversión en recursos y talento, y la existencia de indicadores que miden resultados y comparan instituciones, permiten ofrecer una visión del panorama competitivo, igualmente se requieren herramientas que coadyuven la observación de puntos a favor y en contra, e identifiquen, incluso, aspectos distintivos entre instituciones universitarias.

En este sentido se presenta la MPC aplicada al ámbito de la investigación universitaria. Para construirla, se requiere definir a los competidores y establecer los FCE que una organización de determinado sector debe tener para asegurar éxito en su campo. Para esta propuesta se tomó como referencia un trabajo previo de Suárez y Díaz-Barrios (2014), en el cual se identificaron cuatro factores; tres internos y uno mixto. Los factores internos establecidos fueron: el investigador como protagonista del proceso; la visibilidad, que permite dar a conocer los resultados de las investigaciones a la comunidad científica y a la sociedad en general; y tercero, el modelo de desarrollo, que hace posible direccionar estratégicamente la organización. El factor mixto se refiere a los recursos financieros, los cuales implican tanto la necesidad que tienen las universidades de recibir apoyo por parte del Estado, como también el desafío de generar ingresos propios. Adicionalmente, se desarrollaron veintidós indicadores de desempeño que permiten operacionalizar la puesta en práctica de los FCE.

La inclusión de esta herramienta en la planificación estratégica de la investigación en las instituciones académicas requiere de una visión estratégica por parte de quienes lideran la gestión, dado que supone un cambio de paradigma frente a la incertidumbre del entorno, al tomar una actitud proactiva. Otra bondad de la herramienta es orientar las instituciones permanentemente al éxito, destinando los recursos hacia los factores clave que garanticen objetivos de liderazgo regional en investigación y visibilidad mundial. Adicionalmente, tienen la opción de adoptar y adaptar las mejores prácticas de aquellas otras universidades que sean superiores en un determinado factor. Se espera, entonces, que esta propuesta resulte útil para instituciones universitarias con fortalezas en investigación e interesadas en proyectar su gestión hacia estadios superiores. La investigación necesita guías que la conduzcan en el camino a la excelencia, y esta herramienta que hoy se presenta tiene como objetivo ayudar a construir ese camino. 


\section{Sobre las autoras}

Wendolin Suárez Amaya. Doctora en Ciencias Sociales, con mención en Gerencia; magíster en Gerencia Pública; licenciada en Administración. Profesora-investigadora de la Facultad de Ciencias Empresariales de la Universidad Arturo Prat, Chile. Profesora-investigadora de la Facultad de Ciencias Económicas y Sociales (FCES) de la Universidad del Zulia (LUZ), Venezuela. Investigadora del Centro de Estudios de la Empresa de FCES-LUz, adscrita a las líneas de investigación de gestión universitaria (desde 2001) y modernización empresarial (desde 2013). Miembro de la Red de Investigación Gobernanza Universitaria en Iberoamérica. Investigadora responsable o corresponsable en once proyectos de investigación (2004-2020). Sus áreas de interés son: gestión estratégica, gobernanza universitaria, gestión del talento humano, gestión de la investigación. En 2020, publicó los capítulos de libros: "Investigación según indicadores y rankings. ¿Cómo está Latinoamérica?”; “Formando administradores desde la competencia investigativa para una sociedad postmoderna”; y el artículo de revista "Erratas incurridas en la presentación de artículos en revistas indexadas iberoamericanas de las áreas de ciencias sociales y multidisciplinarias”.

Jazmín Díaz-Barrios. Posdoctora en Investigación en Gerencia; doctora en Ciencias Gerenciales; especialista en Economía Empresarial; licenciada en Ciencias Administrativas. Profesora titular de la Facultad de Ciencias Económicas y Sociales (FCES) de la Universidad del Zulia. Docente (pre- y posgrado) y tutora de tesis. Investigadora del Centro de Estudios de la Empresa de FCES-LUZ, adscrita a las líneas de investigación de gestión universitaria y modernización empresarial. Miembro de la Red de Investigación Gobernanza Universitaria en Iberoamérica. Responsable o corresponsable en catorce proyectos de investigación (2001-2020). Sus áreas de interés son: planificación y control de la gestión organizacional, sostenibilidad de las organizaciones, gobernanza. En 2020, publicó los capítulos de libros: "Formando administradores desde la competencia investigativa para una sociedad postmoderna"; "Políticas de generación de recursos financieros en la universidad pública venezolana"; "Gobierno en la Universidad del Zulia: la injerencia de los grupos sindicales"; "Investigación según indicadores y rankings. ¿Cómo está Latinoamérica?”.

Morela Pereira Burgos. Doctora en Ciencias Sociales, con mención en Gerencia; magíster en Gerencia de Empresas; licenciada en Administración. Profesora titular en la Facultad de Ciencias Económicas y Sociales (FCES) de la Universidad del Zulia. Investigadora del Centro de Estudios de la Empresa de FCES-LUZ, adscrita a las líneas de investigación de gestión universitaria (desde 2001) y modernización empresarial (desde 2013). Miembro de la Red de Investigación Gobernanza Universitaria en Iberoamérica de la Universidad de Los Lagos. Responsable o corresponsable en once proyectos de investigación (2001-2020). Sus áreas de interés son: procesos administrativos, teoría administrativa, liderazgo, planificación estratégica, proyectos de tesis. En 2020, publicó los capítulos de libros: "Formando administradores desde la competencia investigativa para una sociedad postmoderna"; "Gobierno en la Universidad del Zulia: la injerencia de los grupos sindicales"; y el artículo de revista "Estrategias de sostenibilidad en ciudades fronterizas afectadas por la migración. El caso de la ciudad de Maracaibo”. 


\section{Referencias}

Ballbé, M. (1999). La competencia entre universidades. En Ll. Cases (Ed.), Anuario de la competencia 1998 (pp. 177-190). Fundación ICo. Marcial Pons Ediciones Jurídicas y Sociales, S. A. https://anuariocompetencia.fundacionico.es/files/1998/1998.pdf

Beltrán, J. M. (1998). Indicadores de gestión. Herramientas para lograr la competitividad (3. ${ }^{\mathrm{a}}$ ed. Temas Gerenciales). 3R Editores.

Blanco, F. (2000). El control integrado de gestión. Editorial Limusa, S. A.

Camisón, C. y Dalmau, J. (2009). Introducción a los negocios y su gestión. Pearson.

Canales, A. (2011). El dilema de la investigación universitaria. Perfiles Educativos, 33 (especial), 34-44. https://www.iisue.unam.mx/perfiles/articulo/2011-e-el-dilema-de-la-investigacion-universitaria.pdf

Choi, J. I. y Park, C. G. (2013). The key success factors of university entrepreneurship education: Implication from USA university cases. Asia-Pacific Journal of Business Venturing and Entrepreneurship, 8(3), 85-96.

Comisión Europea. (2019). Germany. Higher Education Funding. https://eacea.ec.europa.eu/ national-policies/eurydice/content/higher-education-funding-31_en

Connell, R. (2019). The good university. What universities actually do and why it's time for radical change. ZedBooks Ltd.

Corchuelo, C. (2018). Visibilidad científica y académica en la Web 2.0. Información, cultura y sociedad, (38), 77-88. https://doi.org/10.34096/ics.i38.3724

D'Alesio, I. F. (2008). El proceso estratégico: un enfoque de gerencia. Pearson Educación.

David, F. R. (2008). Conceptos de administración estratégica. (11. a ed.). Pearson Educación.

David, F. R. (2011). Strategic management concepts and cases: A competitive advantage approach (13th ed.). Pearson Education Prentice Hall.

David, F. R. (2013). Conceptos de administración estratégica. (14. a ed.). Pearson Educación.

Garrido, F. (2014). Les Pourquoi da la Stratégie. Enseignements de tous les temps pour les cadres du Xxi siècle [Los porqués de la estrategia. Lecciones de todos los tiempos para los administradores en el siglo xxI]. La Penseé, Multidisciplinary Journal. https://www. researchgate.net/publication/277405272_Les_Pourquoi_de_la_Strategie_Enseignements_de_tous_les_temps_pour_les_cadres_du_XXI_siecle

Herbert, S., Redman, Ch. y Speldewinde, Ch. (2018). Sustaining School-University Partnerships: Threats, Challenges and Critical Success Factors. En L. Hobbs, C. Campbell y M. Jones (Eds.), School-based Partnerships in Teacher Education (pp. 39-57). Springer.

Johnson, G., Whittington, R. y Scholes, K. (2011). Exploring corporate strategy: Text and cases (9th ed.). Pearson Education. 
Koontz, H., Weihrich, H. y Cannice, M. (2012). Administración. Una perspectiva global y empresarial (14. ${ }^{\mathrm{a}}$ ed.). Mc Graw Hill.

López-Leyva, S. (2016). Competitividad de la educación superior en cuatro países de América Latina: perspectiva desde un ranking mundial. Revista de la educación superior, 45(178), 45-59.

Luzardo, A. (2019, 4 de diciembre). Entrevistada por C. Hernández en Latinoamérica y su saldo rojo en innovación y propiedad intelectual, para France24. https://www.france24.com/ es/20191204-latinoamerica-innovacion-propiedad-intelectual-patentes

Marcano, D. y Phélan, M. (2009). Evolución y desarrollo del programa de promoción del investigador en Venezuela. Interciencia, 34(1), 17-24. http://ve.scielo.org/scielo. php?script=sci_arttextypid=S0378-18442009000100005

Meléndez, R. (2010). La producción científica en Venezuela: cantidad vs. calidad. Revista Compendium, (25), 55-64. http://www.ucla.edu.ve/dac/compendium/Revista25/Compendium25_Articulo_5.pdf

Mintzberg, H. (2009). Managing. Berrett-Koehler.

Mora, J., Haldane, J., Younger, R., Lostao P. y Simpson, L. (2015). Reputación de universidades. Ediciones Universidad de Navarra.

Ohmae, K. (1982). The Mind of the Strategist. The Art of Japanese Business. McGraw-Hill.

Páez, J. (2010). La investigación universitaria y la formación del profesorado latinoamericano. Aposta. Revista de Ciencias Sociales, (47), 1-38. https://www.redalyc.org/ pdf/4959/495950243004.pdf

Peláez, M. D. (2016). Competitive Profile Matrix as a tool for evaluating strategic performance of Higher Education Institutions. Journal of Strategy and Performance Management, 4(3), 77-92.

Plewa, C., Korff, N. y Baaken, T. (2013). University-industry linkage evolution: An empirical investigation of relational success factors. $R \& D$ Management, 43(4), 365-380.

Rice, M. P., Fetters, M. L. y Greene, P. G. (2010). University-based entrepreneurship ecosystems: Key success factors and recommendations. En M. L. Fetters, P. G. Greene y M. P. Rice (Eds.), The development of university-based entrepreneurship ecosystems: Global practices (pp. 177-196). Edward Elgar Publishing.

Rockart, J. (1979). Chief executives define their own data needs. Harvard Business Review, (3), 81-93. http://hbr.org/1979/03/chief-executives-define-their-own-data-needs/ar/1

Schofield, T. (2013). Critical success factors for knowledge transfer collaborations between university and industry. Journal of Research Administration, 44(2), 38-56.

Scimago Country Rank (2020). Subject Bubble Chart Venezuela. https://www.scimagojr.com/ mapgen.php?maptype=bcycountry=VE

Suárez, W. y Díaz-Barrios, J. (2014). Factores críticos de éxito en la investigación universitaria venezolana. En F. Ganga, J. Abello y J. Quiroz (Eds.), Gobernanza universitaria. 
Aproximaciones teóricas y empíricas (pp. 109-118). Editorial Cedac. https://www.researchgate.net/publication/270889779_Factores_criticos_de_exito_en_la_investigacion_universitaria_venezolana

Thompson, A., Peteraf, M., Strikland A. J.y Gamble, J. (2012). Administración estratégica. Teoría $y$ casos (18. ${ }^{\mathrm{a}}$ ed.). Mc Graw Hill.

Times Higher Education. (2020). THE World University Rankings 2020: methodology. https:// www.timeshighereducation.com/world-university-rankings/world-university-rankings-2020-methodology

Vera, J. y Díaz, R. (2012). ¿Qué es un competidor directo? Estudio para corroborar la percepción de competencia directa con base en tres factores. Contaduría y Administración, 57(1), http://www.scielo.org.mx/scielo.php?script=sci_arttext\&pid =S0186-10422012000100008

Xue, B., Li, M. y Wang, S. (2004). A comparison between Chinese and American Governments in financing the scientific research in higher educational institutes. Tsinghua Journal of Education, (6), 54-59.

Zambrano, A. (2006). Planificación estratégica, presupuesto y control de la gestión pública. Editorial Universidad Católica Andrés Bello.

Zimmerer, T., Scarborough, N. M. y Wilson, D. (2008). Essentials of entrepreneurship and small business management [Fundamentos de la iniciativa empresarial y la gestión de la pequeña empresa] (5th ed.). Prentice Hall. 